\title{
The Mystery of the Melamine Story
}

DOI: $10.1134 / \mathrm{S} 1061934810100011$

The nutritional value of foodstuffs is primarily determined by its protein content. Standard methods for the estimation of the total concentration of proteins are based on the determination of the total concentration of nitrogen. Knowing this, unfair producers of dry feeds for domestic animals in the United States and also producers of dried milk and milk-based baby food in China utilized the lack of specificity of analytical methods and began to add melamine to these products. In this compound, a small molecule includes six nitrogen atoms (three in a six-membered heterocycle with conjugated bonds and three in amino groups bound to carbon atoms of the heterocycle). This compound is used in the production of some fertilizers, plastics, and laminates. Melamine is almost nontoxic, and the falsifiers felt that they had nothing to fear.

However, about a thousand cats and dogs fed with dry feed containing post detected melamine died in 2007 in the United States. Many domestic animals became ill. In 2008, six children died in China (and about 200 thousand suffered); they had eaten baby food on the basis of dried milk in which melamine was also discovered. A scandal broke after these events in China.

Researchers in the United States promptly understood that melamine, an indifferent substance in itself, reacted in an organism with its analogue, cyanuric acid, which was present in melamine as an impurity. It was believed that this reaction results in a crystalline substance affecting the kidneys. However, the Chinese dried milk contained pure melamine, and the World Health Organization then revised its opinion on the behavior of melamine in an organism. It is believed that melamine reacts with uric acid contained in urine, especially in babies. The complex formed, melamine urate, blocks the kidney; it is a type of kidney stone. If there is a lot of melamine, the kidney can fail.

In 2007, the necessity of developing analytical methods allowing the prompt and safe detection of melamine in foodstuffs became an urgent task. It was a challenge for analysts. We can note with satisfaction that many efficient methods have been developed within a short period of time.

The first methods developed under the aegis of the US Food and Drug Administration (FDA), were based on gas or, more accurately, liquid chromatography with mass spectrometric detection. This was, of course, a natural way to solve the problem. Such methods ensure sufficient sensitivity (it was necessary that the detection limit was not worse than $10^{-4} \%$ ), but are sufficiently long and require reliable equipment.

Attempts were also made to use immunochemical methods (ELISA), but it was found these suffer from interference in the analysis of complex matrices. The use of IR spectrometry in the near and mid-IR regions is more promising. Some of the proposed methods ensure the required detection limits. A drawback is the necessity of recalibration in going from one matrix to another.

It seems that today the most efficient method is direct mass spectrometry with atmospheric pressure plasma ionization. The serial DART mass spectrometer from Japanese JEOL Corporation allows the determination of melamine and its analogues within several seconds with no sample preparation.

One can read about this in greater detail in the paper by S.L. Rovner [1], which I used in the preparation of this note. There, one can also find references to original publications on the methods mentioned above.

I would also like to add that the concentration of melamine must, probably, be controlled not only in foodstuffs, but also in drugs, which also contain nitrogen compounds [2].

\section{REFERENCES}

1. Rovner, S.L., Chem. Eng. News, 2009, May 25, p. 36.

2. Chem. Eng. News, 2009, August 17, p. 27.

Yu. A. Zolotov 